\title{
A STUDY ON THE ANTIDEPRESSANT EFFECT OF DANZHI XIAOYAO POWDER
}

\author{
Zheng Lun* \\ Youth \& Children Research Institute, China Youth University for Political Sciences, Beijing 100089, China \\ *inrgne154@126.com
}

\begin{abstract}
Background: Danzhi Xiaoyao Powder is used for regulating qi and blood, soothing liver-qi stagnation and adjusting the function of internal organs in the clinical. The objective of this paper was to study the antidepressant effect of Danzhi Xiaoyao Powder.

Methods: Experimental animals were grouped and given different doses of xiaoyao powder and Fluoxetine Hydrochloride tablets respectively. Mouse models of behavioural despair such as tail suspension test and forced swimming test were used to evaluate the antidepressant effect of Xiaoyao. Results: Danzhi Xiaoyao Powder could shorten the immobility time of mice in the tail suspension and forced swimming despair models, and had a significant effect on them, while having no significant effect on locomotor activity in mice.

Conclusion: Danzhi Xiaoyao Powder has a good antidepressant effect. The material basis and physiological and biochemical mechanisms of its action should be determined by further study.
\end{abstract}

Keywords: Danzhi Xiaoyao Powder; anti-depression; tail suspension test; forced swimming test

\section{Introduction}

Song Dynasty's "Formulary of Peaceful Benevolent Dispensary" recorded that Xiaoyao Powder consisted of Radix Bupleuri, Radix Angelicae Sinensis, Radix Paeoniae Alba, Poria cocos, Rhizoma Atractylodis Macrocephalae and Radix Glycyrrhizae Preparata. On this basis, Cortex Moutan and Fructus Gardeniae are added to constitute the Danzhi Xiaoyao Powder, which is used clinically for regulating qi and blood, soothing liver-qi stagnation and adjusting the function of internal organs (Liu et al., 1998). This study observed the antidepressant effect of Danzhi Xiaoyao Powder by the locomotor activity test, tail suspension test, and forced swimming test in mice.

\section{Materials and Methods}

Drugs

The drug included Danzhi Xiaoyao Powder which consisted of Cortex Moutan, Fructus Gardeniae, Radix Bupleuri, Radix Angelicae Sinensis, Radix Paeoniae Alba, Poria cocos, Rhizoma Atractylodis Macrocephalae and Radix Glycyrrhizae Preparata. The above herbal medicines were formulated according to the original proportion, and decocted twice with a 10-fold volume of water. Filtrates were combined, evaporated, prepared into extract powder, and then placed in the dryer for later use. Immediately before use, the powder was prepared to the desired concentration with distilled water. Also used was Fluoxetine Hydrochloride Tablets, Changzhou Siyao Pharmaceuticals Co., Ltd., batch number: 000530.

\section{Animals}

Kunming mice, male, weighing 20-24 g, were provided by the China Medical University. The mice were kept indoors at $20 \pm 1^{\circ} \mathrm{C}$ in separate cages, and were fed and watered ad libitum. The all experimental animals were permitted by the Beijing Animal Research Ethics Committee.

Mice were randomised into five groups, namely the Danzhi Xiaoyao Powder high-, medium-, and low-dose groups, positive control group (60 mg/kg fluoxetine hydrochloride), and blank control group (distilled water). The mice in each group were intragastrically administered once daily at 8:00 am for 7 consecutive days, and the tests were performed on the 7 th day $1 \mathrm{~h}$ after administration. 
http://dx.doi.org/10.4314/ajtcam.v11i1.32

\section{Open field test}

Referring to the method of Soubrie P, et al. ( $\mathrm{Li}, 1991), 1 \mathrm{~h}$ after the last intragastric administration, the mice were placed in a cylindrical container $30 \mathrm{~cm}$ in diameter and $20 \mathrm{~cm}$ in height with bottom divided into 19 equal sections. The walking routes and the number of grid crossings of mice within $2 \mathrm{~min}$ in each group were compared.

Tail suspension test in mice (Steru et al., 1985)

Mice were fastened to the edge of a steel stand by the adhesive tape placed $2 \mathrm{~cm}$ from the tip of the tail, so that they were suspended upside-down in a box $(40 \mathrm{~cm} \times 40 \mathrm{~cm} \times 30 \mathrm{~cm})$ with their heads $6 \mathrm{~cm}$ above the box bottom. Interference with each other was avoided, and certain distance was kept between mice of each experimental group. At the beginning, mice moved up and down instinctively attempting to find a climbing site. After a period of time, activity decreased due to disappointment, that is, the occurrence of intermittent immobility. Stopwatch was started when the mice were moving, observation was continued for 6 min, and the immobility time of mice within the last 4 min was calculated.

Forced swimming test in mice (Porsolt et al., 1977; Nixon, 1994)

$30 \mathrm{~min}$ after the last administration, the mice were placed in a circular glass jar $(25 \mathrm{~cm}$ high $\times 15 \mathrm{~cm}$ diameter $)$ filled with water to a depth of $15 \mathrm{~cm}$, and maintained at $25^{\circ} \mathrm{C}$. Each jar contained one mouse. After 2 min of swimming, observation and timing began. The observation continued for $4 \mathrm{~min}$, and the immobility (the state in which the mouse stops struggling in the water, or remains floating, making only minimum movements of limbs) time of mice within this 4 min was accumulated.

\section{Statistical processing of data}

ANOVA and t-test on the data of experimental results were performed using SPSS 17.0 statistical software. P $<0.05$ indicated that the difference was statistically significant.

\section{Results \\ Open field test}

The experimental results are shown in Table 1. The locomotor activity in mice administered with Danzhi Xiaoyao Powder did not change significantly compared with the blank control group, indicating that the drug had no significant effect on locomotor activity in mice.

Table 1: Effect of Danzhi Xiaoyao Powder on locomotor activity in mice $(\bar{x} \pm s)$

\begin{tabular}{lccc}
\hline \multicolumn{1}{c}{ Group } & Dose $(\mathrm{g} / \mathrm{kg})$ & Number of animals & Locomotor activity within 5 min (s) \\
\hline Control group & & 10 & $148.45 \pm 25.38$ \\
Fluoxetine hydrochloride group & 0.06 & 10 & $135.54 \pm 26.98$ \\
Danzhi Xiaoyao Powder & 5 & 10 & $127.24 \pm 27.64$ \\
Danzhi Xiaoyao Powder & 10 & 10 & $130.45 \pm 30.48$ \\
Danzhi Xiaoyao Powder & 20 & 10 & $134.21 \pm 35.68$ \\
\hline
\end{tabular}

\section{Effect on tail suspension test in mice}

The experimental results are shown in Table 2. Compared with the blank control group, high and medium doses of the decoction significantly shortened the immobility time of mice in the tail suspension test $7 \mathrm{~d}$ after the administration. Low dose reduced the immobility time in the tail suspension test in mice to some extent, but the difference was not significant compared with the blank control group. 
http://dx.doi.org/10.4314/ajtcam.v11i1.32

Effect of Danzhi Xiaoyao Powder on immobility time of mice in the forced swimming test

The experimental results showed that each Danzhi Xiaoyao Powder dose group could evidently shorten the immobility time of mice in the forced swimming test, and the differences were significant compared with the blank control group. The results are shown in Table 3.

Table 2: Effect of Danzhi Xiaoyao Powder on immobility time of mice in the tail suspension test $(\bar{x} \pm \mathrm{s})$

\begin{tabular}{cccc}
\hline Group & Dose $(\mathrm{g} / \mathrm{kg})$ & Number of animals & $\begin{array}{c}\text { Immobility time } \\
\text { within 6 min }(\mathrm{s})\end{array}$ \\
\hline Control group & & 10 & $112.58 \pm 46.57$ \\
Fluoxetine hydrochloride group & 0.06 & 10 & $64.51 \pm 12.62^{* *}$ \\
Danzhi Xiaoyao Powder & 5 & 10 & $99.64 \pm 12.39$ \\
Danzhi Xiaoyao Powder & 10 & 10 & $81.48 \pm 21.65^{*}$ \\
Danzhi Xiaoyao Powder & 20 & 10 & $66.35 \pm 14.74^{* *}$ \\
\hline
\end{tabular}

Comparison with the control group $* \mathrm{P}<0.05$, ** $\mathrm{P}<0.01$ (the same below)

Table 3: Effect of Danzhi Xiaoyao Powder on immobility time of mice in the forced swimming test $(\mathrm{x} \pm \mathrm{s})$

\begin{tabular}{lccc}
\hline \multicolumn{1}{c}{ Group } & Dose $(\mathrm{g} / \mathrm{kg})$ & Number of animals & Immobility time within 4 min $(\mathrm{s})$ \\
\hline Control group & & 10 & $99.12 \pm 10.65$ \\
Fluoxetine hydrochloride & 0.06 & 10 & $68.47 \pm 12.68^{*}$ \\
group & 5 & 10 & $90.57 \pm 10.35$ \\
Danzhi Xiaoyao Powder & 10 & 10 & $91.74 \pm 14.78$ \\
Danzhi Xiaoyao Powder & 20 & 10 & $67.59 \pm 10.34 *$ \\
Danzhi Xiaoyao Powder & & & \\
\hline
\end{tabular}

\section{Discussion}

Depression is a major type of mood disorder (Hou et al., 2007; Wang et al., 2007), which is a syndrome mainly characterised by a significant and lasting low mood. Its main manifestations include emotional downturn, reduced verbal action, retardation of thinking, slowness of movement, frequent self-blame, self-guilt, and even attempted suicide. With the accelerated pace of life, the pressures from various aspects of society continue to increase. Depression has become a common disease of modern society with a high incidence; its incidence is also rising continually. According to statistics, depressive illness ranks fourth among the disabling diseases worldwide. It is estimated that in 2020, its incidence will further rise, which will rank second only to the number one disease - ischemic heart disease (He et al., 2003). Modern pharmacological studies have shown that pathogenic factors leading to depression are very complex and that there are many causes for its induction. At present, pharmaceutical studies on the prevention and treatment of depressive illness are carried out mainly around the monoamine neurotransmitter norepinephrine (NE) in the brain, 5-hydroxytryptamine (5-HT), etc.

From the point of view of traditional Chinese medicine theory, the causes of depression are mostly due to extreme changes of emotions and internal emotional injuries led by anxiety, frustration, mental stress, sorrow, worry, fear, etc. Its pathogenesis lies in two aspects: heart-qi deficiency and liver-qi depression. Although emotional injury is an important cause of depression, but whether it can make the patients reach the depressive degree is closely related to the factors such as whether the personality is introverted, whether the physical constitution is weak. Clinically, depression is treated by soothing-liver, regulating qi, and relieving liver depression in TCM, accompanied by therapies such as heart and spleen invigoration, liver and kidney nourishment, and mind tranquilisation by heart nourishment.

In this study, starting from the effective prescription for clinical treatment of depression, the antidepressant pharmacological effects of Danzhi Xiaoyao Powder was preliminarily investigated, and the antidepressant effect of this prescription was confirmed, laying the foundation for in-depth exploration of its mechanism of action. The mouse models of forced swimming and tail suspension adopted in this study are sensitive to 
http://dx.doi.org/10.4314/ajtcam.v11i1.32

most antidepressant drugs, which is a classic screening model for antidepressants. In addition, this study also measured the locomotor activity of mice. The methods were simple, fast, effective and intuitive, further improving the reliability of the experiment.

The experimental results showed that three Danzhi Xiaoyao Powder dose groups and the fluoxetine hydrochloride control group had no significant effect on locomotor activity in mice, indicating that Danzhi Xiaoyao Powder cannot cause nervous system stimulation within the dose range of 5-20 g/kg. Each treatment group could evidently antagonise the desperate behaviours in two "behavioral despair" models in mice, and apparently shorten the immobility time. Moreover, a good dose-effect relationship was noted between three Danzhi Xiaoyao Powder doses, of which Danzhi Xiaoyao Powder high-dose group showed relatively significant antidepressant effect. Danzhi Xiaoyao Powder, within a dose range not affecting the locomotor activity, could apparently shorten the immobility time in the forced swimming and tail suspension tests in mice, indicating that Danzhi Xiaoyao Powder has an apparent antidepressant effect on mouse models of "behavioral despair".

In conclusion, Danzhi Xiaoyao Powder can obviously improve the depressive state behaviours in mice. The mechanism of its antidepressant effect should be further studied. The antidepressant active constituents or compounds in the prescription should be further identified, and the antidepressant material basis should be further investigated, in order to provide the guarantee for effective clinical diagnosis and treatment of depression.

\section{References}

1. He Yong-mei, Du Shao-li. (2003) Advancement in research on antidepressant TCM. Hunan Guiding Journal of Traditional Chinese Medicine and Pharmacology, 9 (6): 71-73

2. Hou Lin, Li Yan-hua, Wang Jing-jun. (2007) New advances in the study of antidepressants. Medical Recapitulate, 13 (19): $1511-15116$

3. Li Yi-kui. (1991) Experimental Methodology of TCM Pharmacology. Shanghai: Shanghai Scientific \& Technical Publishers, $329-331$

4. Liu Yue, Gu Wei. (1998) Professor Huang Huang's experience in application of Banxia Houpu Decoction. Forum On Traditional Chinese Medicine, 13 (4): 24-25

5. Nixon MK. (1994) Additive effects of Lithium and antidepressants in the forced swimming test: further evidence for involvement of the serotoninergic system. Psychopharmacology, 115: 59-64

6. Porsolt RD, Bertin A and Jalfre M. (1977) Behavioral despair in mice:a primary screening test for antidepressants. Arch IntPharmacodyn Ther, 229: $327-336$

7. Steru L, ChermatR, Thierry B. (1985) The tail suspension test: a new method for sceening antidepressants inmice. Psychopharmaco, 85(3): 367-370

8. Wang Xiao-qin, He Ming-hua, Wang Qin. (2007) Overview of development of antidepressant drugs. Capital Medicine, 11: $33-34$ 